\title{
THE SPECTRAL THEORY OF POSETS AND ITS APPLICATIONS TO $C^{*}$-ALGEBRAS
}

\author{
BY
}

\author{
A. H. DOOLEY( ${ }^{1}$ )
}

ABSTRACT. This paper uses methods from the spectral theory of partially ordered sets to clarify and extend some recent results concerning approximately finite-dimensional $C^{*}$-algebras. An extremely explicit description is obtained of the Jacobson topology on the primitive ideal space, and it is shown that this topology has a basis of quasi-compact open sets. In addition, the main results of [4] are proved using only elementary means.

Introduction. Bratteli [3] introduced the idea of an approximately finitedimensional $C^{*}$-algebra (or AF $C^{*}$-algebra)-one which is the limit of an inductive system of finite-dimensional $C^{*}$-algebras. In particular, [3] gives an analysisof the primitive ideals of an AF $C^{*}$-algebra, relating them to certain subsets of the diagram of $A$-a subset of $N \times N$ equipped with a binary relation, $\downarrow$. In [4], this formalism is used to give some topological results on the space Prim A of primitive ideals of $A$, equipped with the Jacobson topology. Bratteli notices that the diagram may be considered a partially ordered set, but makes no real use of this fact; his topological results are mostly proved by recourse to the ideal theory of $C^{*}$-algebras.

In this paper, I work entirely within the framework of the theory of partially ordered sets, directly deducing topological results about Prim A. Use of a notion of spectrum for a partially ordered set (related to that of [6]) enables one to give an extremely explicit description of the Jacobson topology on Prim A. Given this description, I am able to reduce the proofs of the main results on spectral theory in [4] to exercises in elementary set theory. The paper not only adds new methods, but also new results. For example, I show that Prim A has a basis of quasi-compact open sets. Bratteli [5] has since given a different proof of this result based on functional analytic methods.

This seems to be one of the very few classes of $C^{*}$-algebras where, in the

Received by the editors October 3, 1974 and, in revised form, June 17, 1975. $06 A 10$.

AMS (MOS) subject classifications (1970). Primary 46L05, 46L25; Secondary

( $\left.{ }^{1}\right)$ The author was supported during part of this research by a French Government Scholarship at Université Paris VI. 
absence of Hausdorff separation for Prim A, a very explicit description of the Jacobson topology is available. (Another is considered in [1].) The methods of this paper may have applications to algebras which are limits in the category of $C^{*}$-algebras of systems more general than inductive systems.

The paper is organized as follows. $\S 1$ serves to recall some results of [3] and to introduce the Bratteli poset $D(A)$ of an AF $C^{*}$-algebra $A$. $\$ 2$, which contains the bulk of the work, is independent of the other two sections; it deals with the spectral theory of a class of partially ordered sets, a class which includes the Bratteli posets. For each partially ordered set $P$ of this class, I define a topological space Spec $P$ whose topology is explicitly described. A number of results are proved about the topology of Spec $P$. Finally, in $\S 3$, I combine the results of the first two sections by means of a theorem which asserts that Prim $A$ is homeomorphic to Spec $D(A)$, and deduce the main results of the paper.

I would like to thank Professor Karl H. Hofmann for suggesting the ideas behind this research, and for his many helpful comments during its development.

Conventions. If $X$ is a topological space, I shall mean by a basis for the topology of $X$ a set $B$ of open subsets of $X$ such that every open set may be expressed as a union of sets from $B$.

I shall only ever consider one topology on the $C^{*}$-algebra $A$-the norm topology. Thus a closed ideal of $A$ will mean a norm-closed ideal, and so on.

1. Bratteli diagrams and Bratteli posets. I shall begin by recalling some results of [3]. Let $A$ be a $C^{*}$-algebra (with identity), which is the inductive limit of the system $\left(A_{n}, j_{n}\right)$, the $A_{n}$ being finite-dimensional $C^{*}$-algebras, and $j_{n}: A_{n-1}$ $\rightarrow A_{n}$ an embedding. Such an algebra is called an AF $C^{*}$-algebra. One may identify $A$ with $\bigcup_{n=1}^{\infty} A_{n}$. Since any finite-dimensional $C^{*}$-algebra may be uniquely decomposed as a direct sum of $m \times m$ matrix algebras, one can write $A_{n}=\bigoplus_{k=1}^{k} M_{(n, k)}$, where each of the $M_{(n, k)}$ is an $m \times m$ matrix algebra for some $m$. The embedding $j_{n}$ decomposes into its components $j_{(n-1, k),\left(n, k^{\prime}\right)}$ : $M_{(n-1, k)} \rightarrow M_{\left(n, k^{\prime}\right)}$. Bratteli denotes by $D(A)$ the set $\{(n, k) \mid n \in N, k=1$, $\left.\ldots, k_{n}\right\}$, and defines a relation " $\downarrow$ " on $D(A)$ by $(n, k) \downarrow\left(n+1, k^{\prime}\right)$ if $j_{(n, k),\left(n+1, k^{\prime}\right)}$ is nonzero. I shall call the set $\mathcal{D}(\mathrm{A})$ equipped with the relation $\downarrow$, the Bratteli diagram of $A$.

In general, let $D$ be a nonempty subset of $N \times N$ with the property that, for each $n \in N$., $\{m \mid(n, m) \in D\}$ is finite, and $(0, m) \in D$ if and only if $m=0$. Suppose $N$ is equipped with a binary relation $\downarrow$ satisfying sonditions (i), (ii) and (iii) on p. 201 of [3]. Then $(D, \downarrow)$ will be said to be a Bratteli diagram. In [3] it is shown that every Bratteli diagram arises as the Bratteli diagram of $A$ for some AF-algebra A.

(1.1) Definition. Let $(D, \downarrow)$ be a Bratteli diagram. Define another rela- 
tion, " $\geqslant$ " on $D$ as follows: for $x, y \in D, x \geqslant y$ if there exists a finite sequence $\left\{z_{i}\right\}_{i=1}^{r}$ of elements of $D$ with $z_{1}=x, z_{r}=y$, and for each $i=1, \ldots, r-1$, $z_{i} \downarrow z_{i+1}$.

It is easily seen that $(D, \geqslant)$ is a partially ordered set with a maximum element. Let $d: D \rightarrow N$ be the projection of $D$ onto its first factor. I will call $(D, \geqslant)$ the Bratteli poset of $(D, \downarrow), d$ its degree function. It is easy to verify the truth of

(1.2) LEMMA Let $(D, \downarrow)$ be a Bratteli diagram, $(D, \geqslant)$ the associated Bratteli poset with degree function $d$. Then

(i) If $x \leqslant y$ then $d(x) \geqslant d(y)$.

(ii) For all $n \in N, D^{n}:=d^{-1}(n)$ is a finite set.

(iii) For all $x, y \in D$, if $x \geqslant y$ and $x \neq y$, then there exists $z \in D^{d(x)+1}$ with $x \geqslant z \geqslant y$.

(iv) D has no minimal elements.

Conversely, if $(D, \geqslant)$ is any partially ordered set with a maximum element, equipped with a function $d: D \rightarrow N$ satisfying (i)-(iv) above, there exists a Bratteli diagram whose associated Bratteli poset is isomorphic to $(D, \geqslant)$.

Any partially ordered set $(D, \geqslant)$, equipped with a function $d: D \rightarrow N$ satisfying (1.2)(i)-(iv) will be called a Bratteli poset.

I shall require the following

(1.3) Lemma. Let $A$ be an AF $C^{*}$-algebra, $(D(A), \downarrow)$ its Bratteli diagram and $(D(A), \geqslant)$ its Bratteli poset. The following conditions are equivalent:

(i) $A$ is abelian.

(ii) For all $x \in[D(A)]^{n}, n \geqslant 1$, there is a unique $y \in D(A)^{n-1}$ with $y \downarrow x$.

(iii) For all $x \in D(A),\{y \mid y \geqslant x\}$ is a chain.

Proof. The equivalence of (i) and (ii) is given in (3.1) of [4]; the equivalence of (ii) and (iii) is simple verification and is left to the reader.

2. The spectral theory of certain partially ordered sets.

(2.1) Notation. A poset is a set $P$ together with a partial order, $\leqslant$, and a greatest element, 1 .

If $x \in P$, I set $\uparrow x=\{y \in P \mid y \geqslant x\}, \downarrow x=\{y \in P \mid y \leqslant x\}, \underline{x}=\{y \in$ $P \mid y$ is maximal in $\downarrow x \backslash\{x\}\}, \bar{x}=\{y \in P \mid y$ is minimal in $\uparrow x \backslash\{x\}\}$.

If $x, y \in P$, let $x \wedge y=\{z \in P \mid z$ is maximal in $\downarrow x \cap \downarrow y\}, x \vee y=\{z \in$ $P \mid z$ is minimal in $\uparrow x \cap \uparrow y\}$. Some of these sets may be empty.

(2.2) Definition. A poset $P$ is called co-well ordered if

(I) Every chain in $P$ has a maximal element.

$P$ is (weakly) well ordered if 
(II) Every chain in $P$ which is bounded below has a minimal element.

A poset $P$ is called graded if there is a poset morphism $d: P \rightarrow N^{\circ p}$, where $N^{\circ o p}$ is the set of integers with reverse order, such that if $x \leqslant y$ and $d(x)=d(y)$ then $x=y$, and $d(1)=0$.

Thus, for $x \leqslant y, d(x) \geqslant d(y)$. The sets $p^{n}=d^{-1}(n)$ are called the layers of $P$ of degree $n$, and $d$ is called the degree function associated with P. A graded poset is laterally finite if all layers are finite.

The graded poset $P$ with degree function $d$ is said to be well-graded if for all $x \in P, d(x)=\{d(x)+1\}$.

A poset satisfying (I) will briefly be called a $C W$ poset, one satisfying (I) and (II), a WCW poset.

(2.3) Examples. (i) Let $X$ be a set, $P$ the set of all finite subsets under reverse inclusion (i.e. $A \geqslant B$ if $A \subseteq B$ ). Set $d(F)=$ card $F$, for $F \in P$. Then $(P, \geqslant)$ is a well-graded WCW poset which is laterally finite if and only if $X$ is finite.

(ii) Let $M$ be a module over a commutative ring $R$ with $1, P$ the set of finitely-generated submodules under reverse inclusion. If $M$ satisfies the descending chain condition for finitely-generated submodules, then $P$ is a $C W$ poset. If $R$ is a field, then $P$ is a well-graded WCW poset with gradation $d(V)=\operatorname{dim} V$. $P$ is laterally finite if and only if $\operatorname{dim} M<\infty$.

(iii) A partially ordered set $P$ is a Bratteli poset if and only if it is a wellgraded laterally finite poset with no minimal elements.

(2.4) Remarks. (i) Let $S$ be a subset of the CW poset $P$. Then every element of $S$ is majorized by an element which is maximal in $S$. In particular, for $x \in P, \underline{x}=\varnothing$ if and only if $\downarrow x=\varnothing$ if and only if $x$ is minimal in $P$; and for $x, y \in P, x \wedge y=\varnothing$ if and only if $\downarrow x \cap \downarrow y=\varnothing$.

(ii) Let $S$ be a subset of the WCW poset $P$ which is bounded below. Then every element of $S$ is minimized by an element minimal in $S$. Hence, for any $x \in P, \bar{x}=\varnothing$ if and only if $x=1$; and for all $x, y \in P, x \vee y \neq \varnothing$.

[The proofs of (i) and (ii) are straightforward, using Zorn's lemma.]

(iii) Every graded poset is automatically a WCW poset.

(2.5) Definition. A filter in a $\mathrm{CW}$ poset $P$ is a nonempty subset $F$ satisfying the following:

(i) For all $x \in F, \uparrow x \subseteq F$.

(ii) For all $x, y \in F,(x \wedge y) \cap F \neq \varnothing$.

A filter is called recursive if for all $x \in F, \underline{x}=\varnothing$ or $\underline{x} \cap F \neq \varnothing$.

Let $F(P)$ denote the set of all filters in $P$, Spec $\bar{P} \subseteq F(P)$ the set of all recursive filters. A filter which is maximal with respect to inclusion is called an ultrafilter. 
The proof of the following lemma is an easy exercise in elementary set theory, using (2.4)(i) and Definition (2.5).

Lemma. Let $F \in F(P)$. Then

(i) $1 \in F$.

(ii) If $x \in F$ is such that $\underline{x} \cap F=\varnothing$, then $x$ is the smallest element of $F$.

(iii) If $F$ is an ultrafilter, $F$ is recursive.

Topology on Spec $P$. I will always assume that $P$ satisfies at least (I).

For $a \in P$, define $S(a) \subseteq \operatorname{Spec} P$ by $S(a)=\{F \in \operatorname{Spec} P \mid a \in F\}$.

(2.7) Remarks. (i) Let $a, b \in P$. Then $S(a) \cap S(b)=\bigcup_{c \in a \wedge b} S(c)$. [Let $F \in \operatorname{Spec} P$. By 2.5(i) and (ii), $a \in F$ and $b \in F$ if and only if there exists $c \in a \wedge b$ such that $c \in F$.]

(ii) Let $F, G \in \operatorname{Spec} P$. If $F \nsubseteq G$, then there is an $a \in P$ such that $F \in$ $S(a)$ but $G \notin S(a)$.

Thus, the sets $S(a), a \in P$, form a basis for the open sets of a $T_{0}$-topology on Spec $P$. This topology will be called the natural topology and denoted by 0 .

(2.8) Let $X$ be a topological space. A subset $C$ of $X$ is called irreducible if it is closed and not contained in the union of two proper closed subsets of itself.

Lemma. Let $C \subseteq$ Spec $P$ be o-closed, and set $F_{C}=\bigcup_{F \in C} F$. The following statements are equivalent:

(i) $C$ is irreducible.

(ii) Let $a, b \in P$. Then $C \cap S(a) \neq \varnothing \neq C \cap S(b)$ implies that there exists $c \in a \wedge b$ such that $S(c) \cap C \neq \varnothing$.

(iii) Let $a, b \in P$. Then if there exist $F, G \in C$ with $a \in F, b \in G$ then there exists $H \in C$ with $a \wedge b \cap H \neq \varnothing$.

(iv) $F_{C} \in \operatorname{Spec} P$.

If these conditions are satisfied, then for all $a \in P, F_{C} \in S(a)$ if and only if $S(a) \cap C \neq \varnothing$.

Proof. (i) $\Rightarrow$ (ii) If $C$ does not satisfy (ii), one may choose $a, b$ with $C \cap$ $S(a) \neq \varnothing \neq C \cap S(b)$, but for all $c \in a \wedge b, S(c) \cap C=\varnothing$. By (2.7)(i), the latter statement implies that $[S(a) \cap S(b)] \cap C=\varnothing$. Hence $C \cap[S(a)]^{c} \neq \varnothing$ $\neq C \cap[S(b)]^{c}$ and $\left([S(a)]^{c} \cap C\right) \cup\left([S(b)]^{c} \cap C\right)=C$. Thus $C$ is not irreducible.

(ii) $\Rightarrow$ (iii) $\Rightarrow$ (iv) is trivial.

(iv) $\Rightarrow$ (v) Suppose $F_{C} \in \operatorname{Spec} P$. If $C$ is not irreducible, there exist $a, b$ $\in P$ with $\left([S(a)]^{c} \cap C\right) \cup\left([S(b)]^{c} \cap C\right)=C$ but $[S(a)]^{c} \cap C \neq \varnothing \neq$ $[S(b)]^{c} \cap C$. By the former statement (via 2.7(i)), $S(c) \cap C=\varnothing$ for all $c \in$ $a \wedge b$; by the latter $a \in F_{C}$ and $b \in F_{C}$ which implies $(a \wedge b) \cap F_{C} \neq \varnothing$. This is a contradiction. 
The last statement of the lemma is clear.

THEOREM. For every $C W$ poset $P$, the natural topology on Spec $P$ has the property that every irreducible set is a singleton closure.

Proof. Let $C$ be an irreducible set, and define $F_{C}$ as in the lemma. The lemma assures us that $C$ is in fact the closure of $\left\{F_{C}\right\}$.

Acyclic CW posets.

(2.9) Proposition. Let $P$ be a CW poset. The following statements are equivalent:

(i) For all $x, y \in P$ such that $x \wedge y \neq \varnothing$ either $x \leqslant y$ or $y \leqslant x$.

(ii) For all $x \in P, \uparrow x$ is totally ordered.

(iii) Every filter is totally ordered.

(iv) Every recursive filter is totally ordered.

Proof. (iii) $\Rightarrow$ (ii) $\Rightarrow$ (i) $\Rightarrow$ (iv) is straightforward using (2.5). For (iv) $\Rightarrow$ (iii), note that every filter is contained in an ultrafilter and apply (2.6)(iii).

The CW poset $P$ is called acyclic if it satisfies the equivalent conditions of the above proposition.

(2.10) LEMMA. In an acyclic CW poset $P$ every maximal chain is a recursive filter. If $P$ is further a WCW poset, the converse is also true and Spec $P$ is precisely equal to the set of maximal chains in $P$.

Proof. The first statement follows from (2.6)(iii) and (2.9). For the second, suppose $P$ is an acyclic WCW poset, $F \in \operatorname{Spec} P$. Then $F$ is a chain. If $F$ is not maximal, choose $y \in P \backslash F$ such that $F \cup\{y\}$ is a chain. (2.5)(i) shows that $y$ is a lower bound for $F$, and hence by (II), $F$ has a minimum element $x_{0}$ $\geqslant y, x_{0} \neq y$. By (2.4)(i) and (2.5), $\underline{x}_{0} \cap F \neq \varnothing$, a contradiction.

(2.11) Lemma. ACW poset $P$ is acyclic if

(v) For all $a \in P,[S(a)]^{c}=\bigcup_{b \in[\downarrow a \cup \uparrow a]^{c}} S(b)$.

If $P$ is further a WCW poset, the converse is also true; viz. if $P$ is acyclic, $P$ satisfies (v).

Proof. (v) $\Rightarrow(2.9)$ (iv) Let $F \in \operatorname{Spec} P$. Then for $x, y \in F, S(x) \cap$ $S(y) \neq \varnothing$, so by $(v), \bigcup\left\{S(b) \mid b \in[\uparrow y \cup \downarrow y]^{c}\right\} \nsupseteq S(x)$. Hence $x \in \uparrow y \cup \downarrow y$. The second statement follows from (2.10).

THEOREM. Let $P$ be an acyclic WCW poset. Then the natural topology is a zero-dimensional Hausdorff topology.

Duality theorems for well-graded acyclic posets.

(2.12) Let $X$ be a set. A function $D: X \times X \rightarrow R$ is called an ultrametric if 
(i) For all $x, y \in X, D(x, y) \geqslant 0$; if $D(x, y)=0$ then $x=y$.

(ii) Let $x, y, z \in X$. Then $D(x, z) \leqslant \max (D(x, y), D(y, z))$.

Notice that every ultrametric is a metric.

(2.13) Lemma. Let $P$ be an acyclic well-graded poset with degree function d. There exists an ultrametric $D$ on Spec $P$ with range $\{0\} \cup\left\{2^{-n} \mid n \in N\right\}$ such that the topology induced by $D$ is the o-topology; indeed the sets $S(a)$ with $d(a)=n$ are precisely the $2^{-n}$ balls. Moreover, Spec $P$ equipped with $D$ is a complete metric space.

PRoof. For $F, G \in \operatorname{Spec} P$, let $S_{F, G}=\left\{n \in N \quad \forall k \leqslant n, P^{k} \cap F=P^{k} \cap\right.$ $G$. Since $1 \in F \cap G, S_{F, G} \neq \varnothing$. If $S_{F, G}$ is unbounded then $F=G$. If $F \neq$ $G$, let $m_{F, G}=\max \left[S_{F, G}\right]$. Define $D$ : Spec $P \times \operatorname{Spec} P \rightarrow\{0\} \cup\left\{2^{-n} \mid n \in\right.$ $N\}$ by

$$
\begin{aligned}
D(F, G) & =0, & & \text { if } F=G, \\
& =2^{-m_{F}, G,}, & & \text { if } F \neq G .
\end{aligned}
$$

It is easily checked that $D$ is an ultrametric. Using this definition, one sees that for $F \in \operatorname{Spec} P$ such that

(1) $F \cap P^{n}=\{a\}, \quad B\left(F, 2^{-n}\right):=\left\{G \in \operatorname{Spec} P \mid D(F, G) \leqslant 2^{-n}\right\}=S(a)$.

Conversely, for any point $a \in P^{n}, S(a)$ is a $2^{-n}$-ball. [Choose a maximal chain $F$ with $a \in F$. Then $S(a)=B\left(F, 2^{-n}\right)$.]

Finally, let $\left\{F_{n}\right\}$ be a $D$-Cauchy sequence in Spec $P$. To define $F \in$ Spec $P$ such that $F_{n} \rightarrow F$, notice that for all $k \in N$, there exists $N \in N$ such that if $m, n \geqslant N, D\left(F_{n}, F_{m}\right) \leqslant 2^{-k}$. Let $N_{k}$ be the minimum such $N$, and define the subset $F$ of $P$ by $F \cap P^{k}=F_{N_{k}} \cap P^{k}$. It follows that, for all $k \in$ $N, r \leqslant k$ implies $F \cap P^{r}=F_{N_{k}} \cap P^{r}$. Using this fact, one verifies that $F \in$ Spec $P$, and further that, for $k \in N, D\left(F_{N_{k}}, F\right) \leqslant 2^{-k}$. Hence $F_{n} \rightarrow F$.

We deduce

THEOREM. Let $P$ be an acyclic, laterally countable well-graded poset. Then Spec $P$, equipped with the above metric is a zero-dimensional Polish space.

(2.14) I will now show that, under certain conditions, the natural topology is a locally compact topology.

Let $P$ be a well-graded poset. $x \in P$ is said to be finitely based if, for all $y \in \downarrow x, \underline{y}$ is finite. $P$ is said to be (spectrally) finitely based if every recursive filter contains a finitely based element.

Notice that for any well-graded poset $P, 1$ is finitely based if and only if $P$ has finite layers. 
The proof of the following lemma is left to the reader.

LEMMA. Let $P$ be an acyclic well-graded poset. $a \in P$ is finitely based if and only if $S(a)$ is a compact set in the natural topology.

TheOREM. Let $P$ be an acyclic well-graded poset. Then Spec $P$ is a locally compact space if and only if $P$ is spectrally finitely based. Spec $P$ is compact if and only if $P$ has finite layers.

(2.15) In this paragraph, we prove a converse to Theorem (2.13).

Theorem. Let $X$ be a zero-dimensional Polish space. Then there exists a countable acyclic well-graded poset $P$ with no minimal elements, such that Spec $P$ is homeomorphic to $X$. If $X$ is locally compact (compact), $P$ is finitely based (has finite layers).

Proof. Let $X$ be a zero-dimensional Polish space. I shall assume the reader is familiar with the construction of a sifting, as given in [2, IX, §6.5]. I call a sifting $\left(C_{n}, p_{n}, \varphi_{n}\right)$ open if for any $c \in C_{n}, \varphi_{n}(c)$ is open. A slight modification of the construction of [2, IX, $\S 6.5$, Lemma 3] proves that $X$ has a strict open sifting $\left(C_{n}, p_{n}, \varphi_{n}\right)$. It is then clear that the function $f: L(C) \rightarrow X$ constructed in $[2, \S 6.5]$, is open and hence is a homeomorphism. Let $C=\bigcup_{n=0}^{\infty} C_{n}$. Defining, for $c \in C_{n}$ and $c^{\prime} \in C_{m}, c \geqslant c^{\prime}$ if $n \geqslant m$ and $P_{n m}(c)=c^{\prime}$ makes $C$ into a poset; if we set $d(c)=n$ for $c \in C_{n}$, $C$ becomes a countable well-graded poset with no minimal elements. It is not too hard to see that $\operatorname{Spec} C$ is homeomorphic to $L(C)$. This proves the theorem.

(2.16) Let $Y$ be the topological space of the irrational numbers, and let $K$ be the Cantor space constructed on the interval $[0,1]$. By the information in $[7, \S 33, I], K$ is the compactification of $Y$. Using this fact, together with Theorems 1 and 2 of $[7, \S 32, \mathrm{II}]$ and (2.15), one deduces

Proposition. (i) Let $P$ be an acyclic well-graded poset. Then Spec $P$ is homeomorphic to a closed subspace of $Y$. If $P$ is in addition laterally finite, then Spec $P$ is homeomorphic to a closed subspace of $K$.

(ii) Let $X$ be any Polish spaçe. Then there exists a laterally countable wellgraded poset $P$, with no minimal elements such that $X$ is a quotient space of Spec $P$. If $X$ is, in addition, compact, we may suppose that $P$ is laterally finite.

Acyclic covers.

(2.17) Let $P$ be a $C W$ poset. Denote by $\widetilde{P}$ the set of all totally ordered subsets $T$ of $P$ such that

(i) $T$ has a minimum element $x_{T}$.

(ii) $T$ is maximal amongst the totally ordered subsets with minimum element $x_{T}$. 
Define $p: \widetilde{P} \rightarrow P$ by $p(T)=x_{T}=\min T$. For $T_{1}, T_{2} \in \widetilde{P}$, let $T_{1} \geqslant$ $T_{2}$ if $T_{1} \subseteq T_{2}$. One then has

Proposition. Let $P$ be a $C W$ poset. $\widetilde{P}$, equipped with $\geqslant$ is an acyclic $C W$ poset and $p$ is a surjective poset map. If $P$ is $W C W$ so is $\widetilde{P}$; if $P$ is graded (well-graded), so is $\widetilde{P}$. Further, if $P$ is graded with finite layers, so is $\widetilde{P}$.

The proof of this proposition is elementary, given the following lemma.

LEMma Let $P$ be a CW poset and let $T \in \widetilde{P}$. Then $\underline{T}=\{T \cup\{y\} \mid y \in$ $\underline{p(T)\}}$.

Proof. First notice that any set of the form $T \cup\{y\}, y \in p(T)$ is in $\widetilde{P}$. Thus, we must check that any element of $\downarrow T \backslash\{T\}$ is majorized by an element of $\widetilde{p}$ of this form. Let $S \in \downarrow T \backslash\{T\}$. Then $p(T) \in S$, and $S \cap \downarrow p(T) \backslash\{p(T)\}$ is a nonempty chain. Hence, by (I) it has a maximum, $y$ say. By the maximality of $S, y \in \underline{p(T)}$, and so $T \cup\{y\} \subseteq S$, as required.

Auxiliary topology on Spec $\widetilde{P}$.

(2.18) Since $\widetilde{P}$ is a CW poset, one may of course define the $o$-topology on Spec $\widetilde{P}$. In this paragraph, I will define a second, coarser topology on Spec $\widetilde{P}$.

For $a \in \widetilde{P}$, let $W(a) \subseteq$ Spec $\widetilde{P}$ be defined by $W(a)=\bigcup_{p(T)<a} S(T)$. It is easily seen that $W(a)=\{F \in \operatorname{Spec} \widetilde{P} \mid$ for some $T \in F, T \cap \downarrow a \neq \varnothing\}$. Using this fact, it is easy to prove that for $a, b \in P, W(a) \cap W(b)=\bigcup_{c \in a} \wedge_{b} W(c)$. Thus $\{W(a) \mid a \in P\}$ is a basis for a topology $\tau$ on Spec $\widetilde{P}$. $\tau$ is clearly coarser than $o$.

Proposition. Let $P$ be a $C W$ poset. The following statements are equivalent:

(i) $P$ is acyclic.

(ii) $p: \widetilde{P} \rightarrow P$ is bijective.

(iii) For any $T \in \widetilde{P}, S(T)=\omega(p(T))$.

Proof. (i) $\Rightarrow$ (ii) $\Rightarrow$ (iii) is trivial. Suppose $P$ is not acyclic. By (2.9)(ii), we may find $a \in P$ and two chains $T_{1}, T_{2}$, maximal in $\uparrow a$ with $p\left(T_{1}\right)=p\left(T_{2}\right)=$ $a, T_{1} \neq T_{2}$. Choose ultrafilters $F_{i}$ in $\widetilde{P}$ with $T_{i} \in F_{i}$. One then has $F_{1} \in$ $\omega\left(p\left(T_{2}\right)\right)$ but $F_{1} \notin S\left(T_{2}\right)$.

(2.19) LEMMA. On any topological space $X$, there is an equivalence relation $R_{X}$, given by $x R_{X} y$ if and only if $\{\bar{x}\}=\{\bar{y}\} . X / R_{X}$ is a $T_{0}$ space, in fact it is the left reflection of $X$ into the category of $T_{0}$ spaces. The front adjunction of this reflection is the quotient map $q_{X}: X \rightarrow X / R_{X}$.

Since this lemma is well known and easily proved, I shall omit its proof. Let $P$ be a CW poset and let $R$ be the equivalence relation of the lemma 
applied to the topological space $(\operatorname{Spec} \widetilde{P}, \tau)$. Set $\operatorname{Spec}_{0} \widetilde{P}=(\operatorname{Spec} \widetilde{P}, \tau) / R$.

Define $m$ : Spec $\widetilde{P} \rightarrow 2^{P}$ by $m(F)=\bigcup_{T \in F}[\uparrow p(T)]=\uparrow \bigcup_{T \in F} p(T)=\uparrow \bigcup_{T \in F} T$.

(2.20) LEMMA. Let $F, G \in \operatorname{Spec} \widetilde{P}$. The following statements are equivalent.

(i) For all $a \in P, F \in W(a)$ if and only if $G \in W(a)$.

(i) ${ }^{1}$ For all $a \in P, \cup_{T \in F} T \cap \downarrow a \neq \varnothing$ if and only if $\bigcup_{T \in G} T \cap \downarrow a \neq \varnothing$.

(ii) For all $T_{F} \in F$ and for all $T_{G} \in G$, there exist $T_{F}^{\prime} \in F$ and $T_{G}^{\prime} \in G$ such that $p\left(T_{G}^{\prime}\right) \leqslant p\left(T_{F}\right)$, and $p\left(T_{F}^{\prime}\right) \leqslant p\left(T_{G}\right)$.

(iii) The $\tau$-closure of $\{F\}$ is equal to the $\tau$-closure of $\{G\}$.

The proof, an easy exercise in elementary set theory, is omitted.

Proposition. With the notation of (2.19), for $F, G \in \operatorname{Spec} P, m(F) \in$ Spec $P$, and if $F R G$ then $m(F)=m(G)$.

ProOF. It is clear from the definition of $m$ that $m(F)$ satisfies (2.5)(i) and (ii). To show that $m(F)$ is recursive, let $x \in m(F), \underline{x} \neq \varnothing$. Choose $T \in F$ such that $x \geqslant p(T)$. If $x \neq p(T)$, then $\underline{x} \cap \uparrow p(T) \neq \varnothing$ and we have finished. If $x=$ $p(T)$, Lemma (2.17) implies that $\underline{x} \cap m(F) \neq \varnothing$. The second statement follows from the lemma.

Let $m_{0}: \operatorname{Spec}_{0} \widetilde{P} \rightarrow$ Spec $\widetilde{P}$ be the mapping naturally induced by $m$.

(2.21) A subset $B$ of a filter $F \in F(P)$ is called a basis for $F$ if $B$ is totally ordered, and for any $x \in F$ there exists $b \in B$ with $b \leqslant x$.

Theorem. Let $P$ be a $C W$ poset. Then $m_{0}: \operatorname{Spec}_{0} \widetilde{P} \rightarrow \operatorname{Spec} P$ is a homeomorphism onto the dense set of all $F \in \operatorname{Spec} P$ which have a basis. If $P$ is countable, then $m_{0}$ is a homeomorphism onto Spec $P$.

Proof. Using the definition of $m$, together with Lemma (2.20)(ii), it is easy to see that $m_{0}$ is injective. Since $m=m_{0} \circ q_{(\operatorname{Spec} \tilde{p}, \tau)}$ where $\left.q_{(\operatorname{Spec}} \tilde{p}, \tau\right)$ is the surjection of (2.19), it is clear that im $m=\operatorname{im} m_{0}$. Now suppose $\widetilde{F} \in \operatorname{Spec} \widetilde{P}$. Then $\{p(T) \mid T \in \widetilde{F}\}$ forms a basis for $m(\tilde{F})$. Hence im $m \subseteq$ $\{F \in \operatorname{Spec} P \mid F$ has a basis\}. For the opposite containment, I will use a lemma.

Lemma. Let $P$ be a $C W$ poset, $F \in \operatorname{Spec} P$, and $G$ a maximal totally ordered subset of $F$. For $x \in G$, let $T_{x}=(\uparrow x) \cap G$. Then $T_{x} \in \widetilde{P}$ and $\widetilde{F}=$ $\left\{T_{x} \mid x \in G\right\} \in \operatorname{Spec} \widetilde{P}$.

Proof. Let $x \in G$. Since $G$ is maximal in $F$ and $\uparrow x \subseteq F, \uparrow x \cap G$ is maximal in $\uparrow x$; thus $T_{x} \in \widetilde{P}$. To see that $\widetilde{F} \in F(\widetilde{P})$ is easy, so it remains to see that $\widetilde{F}$ is recursive. By Lemma (2.17), it suffices to show that, for $x \in G, \underline{x}=\varnothing$ or $\underline{x} \cap G \neq \varnothing$. But this is obviously the case, since $F$ is recursive and $G$ is maximal.

Suppose now that $F$ has a basis. By Zorn's lemma, $F$ has a maximal basis 
G. Defining $\tilde{F}$ as in the lemma, one sees that $m(\tilde{F})=F$, and so $F \in \operatorname{im} m$.

Next, let $a \in P, F \in S(a) \neq \varnothing$. Let $C$ be a chain maximal in $F$ and containing $a$. The lemma shows that $\widetilde{F}=\{\uparrow x \cap C \mid x \in C\} \in \operatorname{Spec} \widetilde{P}$, and clearly $m(\widetilde{F})$ $\in S(a)$. Thus the image is dense. To show that $m_{0}$ is a homeomorphism, it will suffice to show that for $a \in P, m[\omega(a)]=S(a) \cap$ im $m_{0}$. In fact, from the definitions of $W(a)$ and $m$, it is clear that for all $a \in P$, and for all $\widetilde{F} \in \operatorname{Spec} \widetilde{P}$, $F \in W(a)$ if and only if $m(\widetilde{F}) \in S(a)$.

To complete the proof of the theorem, let $F=\left\{x_{i} \mid i \in N\right\}$ be a countable filter. Define a basis $\left\{y_{i}\right\}$ for $F$ as follows: $y_{0}=x_{0}$. Suppose $y_{i}$ defined. Then since $F$ is a filter, $\left(y_{i} \wedge x_{i+1}\right) \cap F \neq \varnothing$. Let $y_{i+1}$ be an arbitrary element of this set.

(2.22) LEmma a. Let $P$ be a $C W$ poset, $a \in P$, and choose $T_{a} \in \widetilde{P}$ with $p\left(T_{a}\right)=a$. If $q:$ Spec $\widetilde{P} \rightarrow \operatorname{Spec}_{0} \widetilde{P}$ is the map of (2.19), then $q[W(a)]=$ $q[S(T a)]$.

Proof. One knows that $W(a) \supseteq S\left(T_{a}\right)$. Thus let $F \in W(a)$, and choose $T_{0} \in F$ such that $p\left(T_{0}\right) \leqslant a$. Then the set $\left[\cup_{S \in \downarrow T_{0} \cap F} p(S)\right] \cup T_{a}$ is a chain contained in $m(F)$. Choose a chain $C$ containing it and maximal in $m(F)$. By Lemma (2.21), $G=\{\uparrow x \cap C \mid x \in C\} \in \operatorname{Spec} \widetilde{P}$. Clearly $G \in S\left(T_{a}\right)$, and $m(G)$ $=\bigcup_{x \in C} \uparrow x=m(F)$. By Theorem (2.21), $q(G)=q(F)$. This proves the lemma.

LEMMA b. Let $P$ be a laterally countable well-graded poset and suppose $a \in P$ is finitely based. Then $S(a)$ is quasi-compact.

Proof. Let $T_{a}$ be an element of $\widetilde{P}$ such that $p\left(T_{a}\right)=a$. Combining information from (2.21) and (2.22), one has $m\left(S\left(T_{a}\right)\right)=S(a)$. By Lemmas (2.14) and (2.17), $S\left(T_{a}\right)$ is compact. Thus $S(a)$, being a continuous image of a compact set, is quasi-compact.

(2.23) A topological space is called spectral if

(i) $X$ is $T_{0}$.

(ii) Every irreducible set in $X$ is a singleton closure.

(iii) $X$ has a basis of quasi-compact open sets.

(2.8) and (2.22) now prove:

THEOREM. Let $P$ be a well-graded countable poset which is spectrally finitely based. Then Spec $P$ is a spectral space. If $P$ is laterally finite, Spec $P$ is a quasi-compact spectral space.

3. The structure spaces of $A F$-algebras. Throughout this section, $A$ will denote an $A F C^{*}$-algebra with identity, $D(A)=(D(A), \geqslant)$ its Bratteli poset. 
(3.1) Lemma. Let $\Lambda \subseteq D(A)$. Consider the following conditions on $\Lambda$.

(i) For all $x \in \Lambda, \downarrow x \subseteq \Lambda$.

(ii) For all $x \in D(A)$, if $\downarrow x \backslash\{x\} \subseteq \Lambda$ then $x \in \Lambda$.

(iii) If $x, y \in \Lambda^{c}$ then there exists $z \in \Lambda^{c}$ such that $z \leqslant x$ and $z \leqslant y$.

If $\Lambda$ satisfies (i) and (ii), let $\Lambda^{n}=\Lambda \cap[D(A)]^{n}$, put $I=\cup_{n=1}^{\infty} \bigoplus_{x \in \Lambda^{n}} M_{(x)}$, and let $I_{\Lambda}=\bar{I}$. Then $I_{\Lambda}$ is a norm-closed ideal of $A . \Lambda \rightarrow I_{\Lambda}$ is a bijection of the set of all subsets of $D(A)$ satisfying (i) and (ii) above onto the set of normclosed ideals of $\mathrm{A}$. Let $\Lambda \subseteq D(\mathrm{~A})$ satisfying (i) and (ii). Then $I_{\Lambda}$ is a primitive ideal of $A$ if and only if $\Lambda$ satisfies (iii).

Proof. This follows from Theorems 3.3 and 3.8 of [3].

(3.2) LEmMA. Let $\Lambda \subseteq D(A)$. Then $\Lambda$ satisfies (3.1)(i), (ii) and (iii) if and only if $\Lambda^{c}$ is a recursive filter of $D(A)$.

Combining (3.1) with this lemma, we see that $\beta: F \mapsto I_{F c}: \operatorname{Spec} D \rightarrow$ Prim $A$ is a bijection.

(3.3) LEMMA. $A$ basis for the open sets of the Jacobson topology on Prim $A$ is given by $O_{x}=\left\{\varphi \in \operatorname{Prim} A \mid \varphi \nsupseteq M_{(x)}\right\}, x \in D(A)$.

Proof. It suffices to show that any closed set in Prim $A$ is an intersection of sets of the form $O_{x}^{c}=\left\{\varphi \in\right.$ Prim $\left.A \mid \varphi \supseteq M_{(x)}\right\}$. Recall that the closed sets of Prim $A$ are $C_{I}=\{\varphi \in$ Prim $A \mid \varphi \supseteq I\}$ where $I$ runs through all closed ideals of $A$. Since, for $\varphi \in$ Prim $A, \varphi \supseteq M_{(x)}$ if and only if $\varphi \supseteq$ the ideal generated by $M_{(x)}$, each of the $O_{x}^{c}$ is clearly closed. Let $\bar{I}$ be a closed ideal of $A$, where $I=$ $\bigcup_{n=1}^{\infty} \bigoplus_{x \in \Lambda_{I}^{n}} M_{(x)}$. The closed set $C_{\bar{I}}$ is easily seen to equal $\bigcap_{n=1}^{\infty} \bigcap_{x \equiv \Lambda_{I}^{n}} M_{(x)}$

THEOREM. Let $A$ be an AF C*-algebra with identity, $O(A)$ its Bratteli poset. Then the map $\beta: \operatorname{Spec} D(A) \rightarrow$ Prim $A$ is a homeomorphism.

Proof. One has $\beta[S(x)]=0_{x}$. The theorem follows.

Corollary a. Let $A$ be an abelian $A F C *$-algebra with identity. Then Prim $A$ is a compact zero-dimensional Polish space. Every compact zero-dimensional Polish space arises as Prim A for some AF C*-algebra A.

(2.16), combined with Gelfand-Naimark duality, now shows that any abelian $A F C^{*}$-algebra with identity is a $C^{*}$-quotient algebra of the $\mathrm{AF} C^{*}$. algebra $C(K)$, and that any separable abelian $C^{*}$-algebra with identity is a subalgebra of $C(K)$.

Corollary b. Let A be an AF $C^{*}$-algebra with identity. Then Prim A is a quasi-compact spectral space. 
Corollary a is proved as Proposition 3.1 of [4]. Corollary b is an improvement on the results of [4], since it contains the assertion that Prim $A$ has a basis of quasi-compact open sets. That irreducible sets in Prim $A$ are one point closures is proved as Lemma 4.2 of [4], but the proof is considerably less elementary than the proof of Theorem (2.8). Corollary $b$ has since been proved in [5], by functional analytic methods. [5] also contains a partial converse to Corollary $b$; if $X$ is a spectral space with the additional property that the intersection of two quasi-compact open sets is quasi-compact, then $X$ arises as Prim $A$ for some $A F C^{*}$-algebra $A$.

In conclusion, I remark that, although I have assumed throughout that $A$ has identity, all the results of this paper may easily be generalized to the case where $A$ is without identity. One may associate with $A$ (again via a diagram), a well-graded countable poset, $(D(A), \geqslant)$ which does not necessarily have finite layers, but which does have the property that it is spectrally finitely based. One again has Prim $A$ homeomorphic to Spec $D(A)$, and consequently, Corollary $b$ generalizes to this case.

\section{BIBLIOGRAPHY}

1. H. Behncke and H. Leptin, $C^{*}$-algebras with finite duals, J. Functional Analysis 14 (1973), 253-268. MR 49 \#7790.

2. N. Bourbaki, Elements of mathematics. General topology. Part 2, Hermann, Paris; Addison-Wesley, Reading, Mass., 1966. MR 34 \#5044b; erratum, 40, p. 1704.

3. O. Bratteli, Inductive limits of finite dimensional $C^{*}$-algebras, Trans. Amer. Math. Soc. 171 (1972), 195-234.

4. - Structure spaces of approximately finite-dimensional C*-algebras, J. Functional Analysis 16 (1974), 192-204.

5. - Structure spaces of approximately finite-dimensional $C^{*}$-algebras. II (preprint, 10 pp.)

6. K. H. Hofmann, and K. Keimel, A general character theory for partially ordered sets and lattices, Mem. Amer. Math. Soc. No. 122 (1972). MR 49 \#4885.

7. C. Kuratowski, Topologie. Vol. 1, 3rd ed., PWN, Warsaw, 1952. MR 14, 1000.

DEPARTMENT OF MATHEMATICS, INSTITUTE OF ADVANCED STUDIES, AUSTRALIAN NATIONAL UNIVERSITY, CANBERRA, A.C.T. 2600, AUSTRALIA 